\title{
QUENCHING PROFILES \\ FOR ONE-DIMENSIONAL SEMILINEAR HEAT EQUATIONS
}

\author{
BY \\ STATHIS FILIPPAS AND JONG-SHENQ GUO
}

Institute for Mathematics and its Applications, University of Minnesota, Minneapolis, MN Institute of Applied Mathematics, National Tsing Hua University, Hsinchu, Taiwan 30043, R.O.C.

\begin{abstract}
We are interested in the local behavior, near a quenching point, of a solution of a semilinear heat equation with singular powerlike absorption. Using the method of Herrero and Velazquez, we obtain a precise description of the spatial profile of the solution in a neighborhood of a quenching point at the quenching time, under certain assumptions on the initial data.
\end{abstract}

1. Introduction. In this paper, we consider the problem

$$
\begin{aligned}
& u_{t}=u_{x x}-u^{-\beta} \quad \text { in } \quad(-l, l) \times(0, T), \\
& u(x, 0)=u_{0}(x), \quad x \in[-l, l] \\
& u( \pm l, t)=1, \quad t \in[0, T)
\end{aligned}
$$

where $\beta>0, l>0, T>0$, and $u_{0}(x)$ is smooth and such that $0<u_{0}(x) \leq 1$ and $u_{0}( \pm l)=1$. For any $\beta>0$ fixed, it is well known [20] that there is a finite time $T$ such that the minimum of the solution $u(x, t)$ reaches zero as $t \uparrow T$ for certain choices of $l$ and $u_{0}$. This phenomenon, which is called quenching, has been studied by many authors for the past two decades (see, for example, [12, 20, 21] and references cited therein).

Hereafter we assume that $u$ quenches at finite time $T$. A point $x_{0}$ is said to be a quenching point if there is a sequence $\left\{\left(x_{n}, t_{n}\right)\right\}$ such that $x_{n} \rightarrow x_{0}, t_{n} \uparrow T$, and $u\left(x_{n}, t_{n}\right) \rightarrow 0$ as $n \rightarrow \infty$.

It has been shown $[3,12,15]$ that the set of points at which the solution quenches (at the same time $T$ ) is a finite set and stays a positive distance away from the boundary $|x|=l$. In particular, we have single point quenching if $u_{0}(x)$ has a single minimum and if the solution quenches.

Received July 12, 1991.

1991 Mathematics Subject Classification. Primary 35K20, 35K55, 35K57.

The major part of this work was done while the second author was visiting the Institute for Mathematics and its Applications, University of Minnesota. He thanks the staff of IMA for their hospitality. The research of the second author was partially supported by the National Science Council grant NSC 800208-M-007-09.

The authors thank the referee for his valuable comments.

(C)1993 Brown University 
Concerning the rate at which the solution approaches zero, under the assumption that $u_{0}(x)$ is such that

$$
u_{0}^{\prime \prime}-u_{0}^{-\beta} \leq 0,
$$

the following estimate has been shown $[12,4,14]$ for all values of $\beta>0$ :

$$
\lim _{t \uparrow T}(T-t)^{-1 /(\beta+1)} u(x, t)=(\beta+1)^{1 /(\beta+1)},
$$

uniformly for $\left|x-x_{0}\right|<C(T-t)^{1 / 2}$ for any positive constant $C$. For the corresponding results in higher dimensions, we refer the interested reader to [5, 13].

The above estimate, besides giving the quenching rate, provides us with some information about the asymptotic behavior of $u$ in a space-time parabola (with its vertex at the quenching point) as $t$ approaches $T$. But since the domain of validity of this estimate, tends to zero as we approach the quenching time, any information about the space structure of the solution at the quenching time $T$ is lost. In this work we are interested in learning more about the behavior of $u$ near a quenching point at time $T$.

The similarity between quenching and blowup problems is well known. A typical example of the latter is the following equation:

$$
u_{t}=u_{x x}+u^{p}, \quad-\infty<x<\infty, \quad t>0, \quad p>1,
$$

with continuous, nonnegative and bounded initial conditions. The above equation has been studied extensively in recent years (see for instance, [7-11, 16-19, 22, 23] and references cited therein). Solutions of (1.5), under certain assumptions on the initial data become infinite in finite time. Moreover, an estimate similar to (1.4) holds for the blowup rate.

In a recent series of papers, Herrero and Velazquez [16-19,23] were able to obtain a precise description of the space structure of the solution in a neighborhood of a blowup point. In this work we shall employ these ideas in the study of the quenching problem. Our main result is the following:

Theorem A. Let $u(x, t)$ be the solution of $(1.1)-(1.3)$ which quenches at the point $x_{0}$ at time $T$. Moreover we assume that

(i) $u_{0}(x)$ satisfies $(\mathrm{H})$,

(ii) $0<u_{0}(x) \leq 1$,

(iii) $u_{0}(x)$ has a single minimum.

Then we have that

$$
u(x, T)=\left[\frac{(\beta+1)^{2}}{8 \beta}\right]^{\gamma}\left(\frac{\left|x-x_{0}\right|^{2}}{|\ln | x-x_{0} \mid}\right)^{\gamma}(1+o(1)),
$$

as $\left|x-x_{0}\right| \rightarrow 0$; here $\gamma=1 /(\beta+1)$.

A few remarks are in order. Assumption (i) is essential in our analysis, since the quenching rate estimate (1.4), of which we make strong use, is known under hypothesis (H). In contrast, assumption (ii) (that is, the upper bound) is rather an assumption of technical convenience. We could remove it at the expense of making things slightly more complicated. 
To explain the relevance of (iii) we first have to recall certain facts from [16-19]. It has been shown there, that the space structure near a blowup point at the blowup time $T$, depends on whether there is a single maximum of $u(x, t)$ (for times prior to $T$ ) which becomes infinite at time $T$, or whether two or more maxima coalesce at exactly the blowup time $T$. Moreover, they prove the existence of initial data for which the second possibility happens [17] and they show that such a behavior is of unstable character [19] - the generic one being blowup originating from a single maximum. Returning now to our problem, we expect that a similar situation will hold. The role of assumption (iii) should now be clear: since $u_{0}(x)$ has a single minimum, by standard results (cf. for instance [1]) $u(x, t)$ will have no more than a minimum (in fact exactly one, since we know that it quenches), and therefore the possibility of two or more minima coalescing at time $T$ is ruled out. In analogy with the blowup problem we expect that the coalescence of two or more minima at exactly the quenching time $T$ will result in a behavior different from the one described in (1.6).

One should notice in (1.6) that $u(x, T)$ develops a cusp if $\beta>1$, whereas it is smooth if $0<\beta \leq 1$. This result is not altogether new. It has been shown in [6], under certain assumptions on the initial data, that $u_{x}$ tends to infinity for $\beta>1$ whereas $u_{x}$ tends to zero if $0<\beta<1$.

Our analysis depends heavily on the ideas of $[7,16-18,23]$. Many of the results there are applicable in problem (1.1)-(1.3), either in a straightforward way or after minor modifications. To keep this work at a reasonable size, we simply quote them. On the other hand, several differences appear at the technical level. The relevant arguments are then presented in detail.

The method can be roughly divided into three steps. In the first step, one obtains more information about the asymptotic behavior of the solution in spacetime parabolas, as the quenching time is approached (essentially, by adding one more term in the right-hand side of (1.4)). In the second step, one computes the asymptotic behavior of the solution in slightly larger regions (namely, $\left|x-x_{0}\right|<$ $C \sqrt{(T-t)|\ln (T-t)|})$. This information is then used in the last step where the final time profiles are computed. These steps are presented in Sections 3, 4, 5 respectively, whereas in Section 2 some preparatory material is presented.

2. Preliminaries. In this section we will establish some preliminary results and we will introduce some notation.

At first we note that without loss of generality-as far as the analysis of the present work is concerned-we may assume that $l=1$. As a second step we will replace equation (1.1) by an "extended" one, defined on the whole real line, which of course admits the same solutions with (1.1) when confined in the initial interval $[-1,1]$. This step is of a purely technical character since our main result is clearly of a local nature.

Following [23] we set

$$
V(x, t)=\frac{1}{\sqrt{\pi t}} \exp \left(-\frac{x^{2}}{4 t}\right), \quad W(x, t)=\frac{x}{2 \sqrt{\pi t^{3}}} \exp \left(-\frac{x^{2}}{4 t}\right) .
$$


Clearly $V_{x}=-W, V_{t}=V_{x x}$, and $W_{t}=W_{x x}$ for $x \in \mathbf{R}, t>0$.

To extend (1.1) to the right we define

$$
\bar{u}(x, t)=(x-1) \int_{0}^{t} W(x-1, t-\tau) u_{x}(1, \tau) d \tau+1, \quad x \geq 1 .
$$

One can verify that

$$
\bar{u}_{t}-\bar{u}_{x x}=\bar{g}(x, t), \quad x>1, \quad t>0,
$$

where

$$
\bar{g}(x, t)=2 u_{x}(1,0) V(x-1, t)+2 \int_{0}^{t} V(x-1, t-\tau) u_{x \tau}(1, \tau) d \tau .
$$

Also, we have at $x=1$ :

$$
\begin{aligned}
\bar{u}(1, t) & =1, \quad 0<t<T, \\
\bar{u}_{x}(1, t) & =u_{x}(1, t), \quad 0<t<T .
\end{aligned}
$$

A similar extension can be performed to the left so that finally we get the equation

$$
\tilde{u}_{t}-\tilde{u}_{x x}=f(x, t), \quad x \in \mathbf{R}, 0<t<T
$$

with

and

$$
\tilde{u}(x, t)= \begin{cases}u(x, t), & |x| \leq 1 \\ \bar{u}(x, t), & |x|>1\end{cases}
$$

$$
f(x, t)=\left\{\begin{array}{l}
-u^{-\beta}(x, t), \quad|x| \leq 1 \\
\bar{g}(x, t), \quad|x|>1 .
\end{array}\right.
$$

Notice that $\tilde{u}$ is continuously differentiable at $x= \pm 1$, whereas $f(x, t)$ has a jump discontinuity at $x= \pm 1$. Since the quenching points stay away from the boundary we have (from standard regularity theory) that $u_{x}(1, t)$ and $u_{x t}(1, t)$ are uniformly bounded, whereas under the assumptions of Theorem $A$ they are nonnegative as well. It then follows from (2.1) and (2.2) that, for $|x|>1$,

$$
1 \leq \bar{u}(x, t)<c_{1}<+\infty, \quad 0<\bar{g}(x, t)<c_{2}<+\infty
$$

for suitable constants $c_{1}, c_{2}$. In particular, there are no quenching points for $|x| \geq 1$. From now on we will study the extended equation (2.3).

Assuming that $x_{0}=0$ is a quenching point we next introduce similarity variables:

$$
w(y, s)=(T-t)^{-\gamma} \tilde{u}(x, t), \quad y=x / \sqrt{T-t}, \quad s=-\ln (T-t),
$$

where $\gamma=1 /(\beta+1)$. Then $w(y, s)$ exists for all time $s$ and solves the equation

$$
w_{s}=w_{y y}-\frac{y}{2} w_{y}+\gamma w-F(y, s) \text { in } \mathbf{R} \times \mathbf{R}^{+},
$$

where

$$
F(y, s)= \begin{cases}w^{-\beta}, & |y| \leq e^{s / 2}, \\ -e^{(\gamma-1) s} g(y, s), & |y|>e^{s / 2}\end{cases}
$$

with $g(y, s)=\bar{g}\left(y e^{-s / 2}, T-e^{-s}\right)$. 
By rewriting estimate (1.4) in similarity variables, we have that, under assumption (H) and for any $\beta>0$,

$$
w(y, s) \rightarrow \kappa \equiv \gamma^{-\gamma} \text { as } s \rightarrow \infty, \quad \text { uniformly for }|y|<C .
$$

Moreover, for any $\beta>0$ by [12, Lemma 3.1] there exists a positive constant $B$ such that

$$
w(y, s)>B \quad \text { in } \quad W \equiv\left\{|y|<e^{s / 2}, s>-\ln T\right\} .
$$

Concerning the growth properties of $w$ and its space derivative we have that

- for $\beta>1,|w|<c_{1}|y|+c_{2}$ and $\left|w_{y}\right|<c_{3}$ in $W$,

- for $\beta=1,|w|<c_{4}|y|^{2}+c_{5}$ in $W$,

- for $0<\beta<1,|w|<c_{6}|y|^{2 \gamma}+c_{7}$ and $\left|w_{y}\right|<c_{8} w^{(1-\beta) / 2}$ in $W$,

for suitable positive constants $c_{1}, \ldots, c_{8}$ (see $[12,4,14]$ ). Thus, in all cases, $w$ can grow at most quadratically in $y$. One can easily check that this property is preserved if we replace $W$ by $\mathbf{R} \times \mathbf{R}^{+}$.

To obtain more information about the way $w$ approaches $\kappa$ we next linearize equation (2.7) about $\kappa$. Setting

$$
v(y, s)=w(y, s)-\kappa,
$$

we observe that $v(y, s)$ solves the equation

$$
v_{s}=v_{y y}-\frac{y}{2} v_{y}+v-f(v) \equiv \mathscr{L} v-f(v) \quad \text { in } \quad \mathbf{R} \times \mathbf{R}^{+},
$$

where

$$
f(v)= \begin{cases}(1-\gamma) v-\gamma \kappa+(v+\kappa)^{-\beta}, & |y| \leq e^{s / 2}, \\ (1-\gamma) v-\gamma \kappa-e^{(\gamma-1) s} g(y, s), & |y|>e^{s / 2} .\end{cases}
$$

In the remainder of this section we will discuss some general properties of the equation (2.11). At first we note that the linear operator in (2.11) can be written as $\mathscr{L} v=\frac{1}{\rho}\left(\rho v_{y}\right)_{y}+v$ with $\rho=e^{-y^{2} / 4}$. Let us denote by $L_{\rho}^{2}$ the (Hilbert) space of functions $v$ for which $\int v^{2} \rho<\infty$. The operator $\mathscr{L}$ is easily seen to be a selfadjoint operator in $L_{\rho}^{2}$. Concerning its spectral properties we have the following.

LEMmA 2.1. The eigenvalues of $\mathscr{L}$ are

$$
\lambda_{k}=1-\frac{k}{2}, \quad k=0,1,2, \ldots
$$

The associated orthonormal eigenfunctions are

$$
h_{k}(y)=\alpha_{k} H_{k}(y / 2), \quad k=0,1,2, \ldots
$$

where $H_{k}$ is the $k$ th standard Hermite polynomial and $\alpha_{k}=\left(\pi^{1 / 2} 2^{k+1} k !\right)^{-1 / 2}$.

For a proof see for instance [7]. Concerning the nonlinear term of $(2.11)$ we have Lemma 2.2. Let $f(v)$ be given by (2.12). Then for $s$ large enough

$$
\begin{aligned}
0<f(v) & <C_{1} v^{2}, \\
f(v) & <C_{2}|v|,
\end{aligned}
$$


for suitable constants $C_{1}, C_{2}$ depending only on $B$ and $\beta$.

Proof. Suppose first that $|y|<e^{s / 2}$. Expanding $(v+\kappa)^{-\beta}$ we get

$$
(v+\kappa)^{-\beta}=\kappa^{-\beta}-\beta \gamma v+\frac{1}{2} \beta(\beta+1)(\varphi+\kappa)^{-\beta-2} v^{2},
$$

for some $\varphi$ between 0 and $v$. Therefore

$$
f(v)=c(\varphi, \beta) v^{2}, \quad c(\varphi, \beta) \equiv \frac{1}{2} \beta(\beta+1)(\varphi+\kappa)^{-\beta-2} .
$$

If $v \geq 0$ then $0 \leq c(\varphi, \beta) \leq(\beta / 2)(\beta+1) \kappa^{-\beta-2}=\beta /(2 \kappa)$. If $v<0$, recalling that $B \leq v+\kappa \leq \varphi+\kappa$ we obtain $0 \leq c(\varphi, \beta) \leq(\beta / 2)(\beta+1) B^{-\beta-2}$. Thus (2.13a) has been established. To show (2.13b) we observe that if $v<\kappa$ then $(2.13 \mathrm{~b})$ follows from (2.13a) with $C_{2}=\kappa C_{1}$. If $v>\kappa$ then from (2.12) and the lower bound of $v$ we get that

$$
f(v) \leq(1-\gamma) v-\gamma \kappa+B^{-\beta}<C_{2} v,
$$

for some constant $C_{2}$.

Consider now the case where $|y|>e^{s / 2}$. Then $v(y, s) \geq e^{\gamma s}-\kappa$ (cf. (2.5), (2.6)) whereas $g(y, s)$ is bounded and $(2.13 \mathrm{a}, \mathrm{b})$ follow trivially from $(2.12)$.

For future reference we note that by keeping one more term in the expansion (2.14) one can show in a similar way that

$$
f(v)=\frac{\beta}{2 \kappa} v^{2}+g(v), \quad|g(v)|<c|v|^{3} .
$$

We finally introduce some notation that we are going to use in the next sections. We denote by $\|v\|$ the $L_{\rho}^{2}$ norm of $v$, i.e., $\|v\|=\left(\int v^{2} \rho\right)^{1 / 2}$. We also denote by $v_{+}$ the "unstable" part of $v$, i.e., the projection of $v$ onto the first two eigenfunctions of $\mathscr{L}$ (corresponding to positive eigenvalues), and similarly for $v_{0}$ and $v_{-}$.

3. Refined asymptotics in space-time parabolas. In this section we will study in more detail the large time behavior of $v(y, s)$. More precisely we will show

Proposition 3.1. Given any $C>0$, for $s$ large enough, either

$$
v(y, s)=\frac{\kappa}{2 \beta s}\left(\frac{1}{2} y^{2}-1\right)+o\left(\frac{1}{s}\right),
$$

or else, for some $m \geq 3$ and some constant $c \neq 0$

$$
v(y, s)=c e^{(1-m / 2) s} h_{m}(y)+o\left(e^{(1-m / 2) s}\right),
$$

where convergence takes place in $C^{k}(|y|<C)$ for any $k \geq 0$.

To motivate the above result we now present a formal argument. By discarding the terms of order $O\left(v^{3}\right)$ or higher from the equation satisfied by $v$, we get

$$
v_{s}=\mathscr{L} v-\frac{\beta}{2 k} v^{2} \text {. }
$$

Since the eigenfunctions of $\mathscr{L}$ form a basis for $L_{\rho}^{2}$ we can expand $v(y, s)$ as

$$
v(y, s)=\sum_{k=0}^{\infty} \alpha_{k}(s) h_{k}(y) \text {. }
$$


The presence of a nontrivial null space in the operator $\mathscr{L}$ suggests the use of center manifold theory (see, e.g., [2]). Formally speaking, if a trajectory lies on the center manifold then the dominant mode (for large times $\mathbf{s}$ ) is the neutral one; i.e.,

$$
v(y, s) \sim \alpha_{3}(s) h_{3}(y) \text {. }
$$

Using the above ansatz in Eq. (3.3) we obtain, after some simple calculations, that

$$
\dot{\alpha}_{3}(s)=-\frac{2 \beta c_{2}}{k} \alpha_{3}^{2}+O\left(\alpha_{3}^{3}\right) \text {. }
$$

Solving the above ODE and taking into consideration (3.5) we recover (3.1).

Although this is what should happen generically, there should be "exceptional" solutions of (3.3) which tend to zero exponentially fast. From the center manifold point of view these are trajectories lying on the stable manifold. Such a behavior is described by the second alternative stated in Proposition 3.1.

A similar situation holds for the blowup problem (1.5), for which the corresponding $v$-equation is

$$
v_{s}=\mathscr{L} v+f_{b}(v)
$$

where $\mathscr{L}$ is the same linear operator as in (2.11) and

$$
f_{b}(v)=O\left(v^{2}\right) \text { as } v \rightarrow 0 \text { and }|v(y, s)|<M \text { in } \mathbf{R} \times \mathbf{R}^{+} .
$$

The large-time behavior of the solutions of $(3.7 a, b)$ has been studied in [7] and in a more complete way in [16] where the above Proposition has been established. We should emphasize that a direct application of center manifold ideas, as suggested by our formal argument, is not possible due to the fact that the nonlinear term in (3.7a) (as well as in (3.3)) does not have the required properties in $L_{\rho}^{2}$ (see [7] for details). However, one can make progress by using the special structure of the equation, as well as the fact that we know a priori that our solution tends to zero. We also note that although both approaches $[7,16]$ are clearly influenced by dynamical system ideas, at the technical level they are different.

The main difference between the present situation and problem $(3.7 \mathrm{a}, \mathrm{b})$ is the fact that for problem $(3.7 \mathrm{a}, \mathrm{b}) v(y, s)$ is uniformly bounded in space-time whereas here $v$ may grow (at most) quadratically in $y$. But it turns out that all the arguments used in $[7,16]$ are applicable with minor changes. We now sketch the proof of Proposition 3.1 by combining ideas from both approaches. We begin by recalling some results from [16].

Lemma 3.1. For any $r>1, q>1$, and $L>0$ there exists $s_{0}^{*}=s_{0}^{*}(q, r)$ and $C=C(r, q, L)$ such that

$$
\left(\int v^{r}\left(\cdot, s+s^{*}\right) \rho\right)^{1 / r} \leq C\left(\int v^{q}(\cdot, s) \rho\right)^{1 / q},
$$

for any $s>0$ and any $s^{*} \in\left[s_{0}^{*}, s_{0}^{*}+L\right]$.

Lemma 3.2. Fix $s_{1}>0, A>0$. If for some $b>0$ we have that $\left\|v_{+}\right\|+\left\|v_{-}\right\| \leq b\left\|v_{0}\right\|$ for $s \in\left[s_{1}, s_{1}+A\right]$, then there exists $\theta=\theta(b, A)$ such that

$$
\left\|v\left(\cdot, s_{1}\right)\right\|^{2} \leq \theta\left\|v\left(\cdot, s_{1}+A\right)\right\|^{2} .
$$


LEMMA 3.3. If for any $M>0$, there exists $C=C(M)>0$ such that $\|v\| \leq C e^{-M s}$ for $s>0$, then $v(y, s) \equiv 0$.

These are Lemmas 2.3, 3.1, and 3.5 respectively in [16]. All of them have been proved under the additional assumption that $v(y, s)$ is uniformly bounded. One can check in [16] that this assumption is only used for the derivation of the estimate $\left|f_{b}(v)\right|<c|v|$, which in our case is true (cf. (2.13b)).

As a next step one can show

LEMMA 3.4. Either $\|v\|$ tends to zero exponentially fast or else, for $s$ large enough,

$$
\left\|v_{+}\right\|+\left\|v_{-}\right\|=o\left(\left\|v_{0}\right\|\right) .
$$

This can be proved as in [7] (Theorem A there). Again, the condition that $v$ is bounded is to be replaced by $(2.13 \mathrm{~b})$, the rest of the arguments there staying the same.

We next show

Lemma 3.5. Assume that (3.8) holds. Then

$$
v(y, s)=\frac{\kappa}{2 \beta s}\left(\frac{1}{2} y^{2}-1\right)+o\left(\frac{1}{s}\right),
$$

in the $L_{\rho}^{2}$ sense.

Proof. We present an argument which is simpler than that of [7] or [16]. Let

$$
v_{0}(y, s)=\alpha(s) h_{2}(y), \quad h_{2}(y)=c_{2}\left(\frac{1}{2} y^{2}-1\right), \quad c_{2}=\frac{1}{2} \pi^{-1 / 4} .
$$

We will work with the equation in the form (2.15). Projecting the equation onto $h_{2}(y)$ we get

$$
\begin{aligned}
\dot{\alpha}(s) & =-\frac{\beta}{2 \kappa} \int v^{2} h_{2} \rho+\int g(v) h_{2} \rho \\
& =-\frac{\beta}{2 \kappa} \int v_{0}^{2} h_{2} \rho+\frac{\beta}{2 \kappa} \int\left(v_{0}^{2}-v^{2}\right) h_{2} \rho+\int g(v) h_{2} \rho \\
& \equiv-\frac{\beta}{2 \kappa} \cdot 4 c_{2} \cdot \alpha^{2}+\frac{\beta}{2 \kappa} \mathscr{E}_{1}+\mathscr{E}_{2} .
\end{aligned}
$$

We next estimate $\mathscr{E}_{1}, \mathscr{E}_{2}$. Recalling that $v=v_{+}+v_{0}+v_{-}$we write

$$
\begin{aligned}
\left|\mathscr{E}_{1}\right| & \leq \int\left|v_{+}+v_{-}\right| \cdot\left|v+v_{0}\right|\left|h_{2}\right| \rho \\
& \leq\left(\int\left|v_{+}+v_{-}\right|^{2} \rho\right)^{1 / 2}\left\{\left(\int v^{2} h_{2}^{2} \rho\right)^{1 / 2}+\left(\int v_{0}^{2} h_{2}^{2} \rho\right)^{1 / 2}\right\} \\
& \leq \varepsilon\left(\int v_{0}^{2} \rho\right)^{1 / 2}\left\{c\left(\int v^{4} \rho\right)^{1 / 4}+c\left(\int v_{0}^{2} \rho\right)^{1 / 2}\right\},
\end{aligned}
$$

where we used (3.8) and standard inequalities. Moreover, from Lemmas 3.1 and 3.2 we have

$$
\int v^{4} \rho \leq c\left(\int v^{2}\left(\cdot, s-s^{*}\right) \rho\right)^{2} \leq c\left(\int v^{2} \rho\right)^{2} \leq c\left(\int v_{0}^{2} \rho\right)^{2}=c \alpha^{4}
$$


so that we get $\left|\mathscr{E}_{1}\right|<\varepsilon \alpha^{2}$. By an argument similar to that used in (3.11) we get that $\left|\mathscr{E}_{2}\right| \leq c \alpha^{3}$. So finally we have

$$
\dot{\alpha}=-\frac{2 \beta c_{2}}{\kappa} \alpha^{2}+o\left(\alpha^{2}\right) .
$$

Solving the above ODE and using (3.10) we obtain

$$
v_{0}(y, s)=\frac{\kappa}{2 \beta s}(1+o(1))\left(\frac{1}{2} y^{2}-1\right),
$$

and (3.9) follows since $\left\|v-v_{0}\right\| \leq\left\|v_{+}\right\|+\left\|v_{-}\right\|=o\left(\left\|v_{0}\right\|\right)=o(1 / s)$.

To deal with the case where $\|v\|$ decays exponentially fast one can show

Lemma 3.6. Assume that $\|v\|$ decays exponentially fast. Then either there exists $m \geq 3$ and $C \neq 0$ such that

$$
v(y, s)=C e^{(1-m / 2) s} h_{m}(y)+o\left(e^{(1-m / 2) s}\right),
$$

in the $L_{\rho}^{2}$ sense, or else $v \equiv 0$.

This is Proposition 5.8 in [16]. The same proof carries over here with no changes. We remark that the case $v \equiv 0$ is easily ruled out since it implies that $\tilde{u}(x, t) \equiv$ $(T-t)^{\gamma} \kappa$ (cf. (2.6), (2.10)). Such a solution of (1.1) clearly violates the boundary condition (1.3).

We finally note that once we have obtained convergence in the $L_{\rho}^{2}$ norm one can improve the mode of convergence by using standard interior regularity theory.

4. Asymptotics beyond parabolas. At first we note that under the assumption that $u_{0}(x)$ has a single minimum so do $w(y, s)$ and $v(y, s)$ for all times $s$. It then follows from Proposition 3.1 that the large-time behavior of $v$ is described by (3.1), since any other possibility would imply that $v$ has at least two minima. On the other hand, expansion (3.1) loses its validity in regions where $y^{2} / s \approx 1$. In order to obtain an expansion which is valid in larger regions it is useful to introduce (as in [17]) a new variable $\eta=\sqrt{y} / s=x / \sqrt{(T-t)|\ln (T-t)|}$. Our aim in this section will be to show the following

Proposition 4.1. Under the assumptions of Theorem A, if $x_{0}$ is the (unique) quenching point of (1.1) then, for any $R>0$,

$$
\lim _{t \uparrow T}(T-t)^{-\gamma} u\left(\eta \sqrt{(T-t)|\ln (T-t)|}+x_{0}, t\right)=\kappa\left[1+\frac{\beta+1}{4 \beta} \eta^{2}\right]^{\gamma},
$$

uniformly for $|\eta| \leq R$.

The proof of Proposition 4.1 will be a consequence of a number of lemmas stated and proved below. As a first step we establish the upper bound for the expression in the left-hand side of (4.1). Assuming for simplicity that $x_{0}=0$ we show

LEMMA 4.1. Under the assumptions of Theorem A, we have

$$
(T-t)^{-\gamma} u(\eta \sqrt{(T-t)|\ln (T-t)|}, t) \leq \kappa\left(1+\frac{\beta+1}{4 \beta} \eta^{2}+o(1)\right)^{\gamma},
$$

as $t \rightarrow T$, uniformly for $|\eta| \leq R$. 
Proof. Let

$$
\varphi_{\lambda}(\eta, s)=(T-\lambda)^{-\gamma} u(\eta \sqrt{T-\lambda}, \lambda+s(T-\lambda)), \quad \lambda \in(0, T), s \in(0,1) .
$$

Set $R_{\lambda}=(T-\lambda)^{-1 / 2}$ and let $u_{\lambda}(\eta, s)$ be the solution of the initial boundary value problem:

$$
\begin{aligned}
& u_{\lambda, s}-u_{\lambda, \eta \eta}=0 \quad \text { in } \quad Q_{R_{\lambda}} \equiv\left[-R_{\lambda}, R_{\lambda}\right] \times[0,1] \\
& u_{\lambda}\left( \pm R_{\lambda}, s\right)=(T-\lambda)^{-\gamma}+(T-\lambda)^{1-\gamma} \\
& u_{\lambda}(\eta, 0)=\varphi_{\lambda}(\eta, 0)+(T-\lambda)^{1-\gamma} .
\end{aligned}
$$

Consider the function:

$$
\Phi_{\lambda}(\eta, s)=\left\{u_{\lambda}^{\beta+1}(\eta, s)-(\beta+1) s\right\}^{\gamma} \quad \text { in } \quad Q_{R_{\lambda}} .
$$

Straightforward calculations show that

$$
\begin{aligned}
& \Phi_{\lambda, s}-\Phi_{\lambda, \eta \eta} \geq-\Phi_{\lambda}^{-\beta} \text { in } Q_{R_{\lambda}}, \\
& \Phi_{\lambda}(\eta, 0)>\varphi_{\lambda}(\eta, 0) \text { in }\left[-R_{\lambda}, R_{\lambda}\right], \\
& \Phi_{\lambda}\left( \pm R_{\lambda}, s\right) \geq \varphi_{\lambda}\left( \pm R_{\lambda}, s\right) \text { for } s \in[0,1] .
\end{aligned}
$$

We conclude by comparison that

$$
\Phi_{\lambda}(\eta, s) \geq \varphi_{\lambda}(\eta, s) \quad \text { in } \quad Q_{R_{\lambda}}
$$

for $\lambda$ fixed.

From the definitions of $u_{\lambda}$ and $\varphi_{\lambda}$ it follows that $\left|u_{\lambda}(\eta, 0)\right|<C(T-\lambda)^{-\gamma}$ for some $C$ positive. Arguing as in [23, Lemma 3.7] we get that

$$
u_{\lambda}(\eta, s)=S(s)\left\{\varphi_{\lambda}(\eta, 0)+(T-\lambda)^{1-\gamma}\right\}+O\left(e^{-1 /[16(T-\lambda)]}\right),
$$

uniformly for $|\eta|<\theta<R_{\lambda} / 2,0 \leq s \leq 1$, where $S(s)$ denotes the heat semigroup on the whole real axis.

From (3.1) it follows that

$$
\varphi_{\lambda}(\eta, 0)=\kappa+\frac{\kappa}{2 \beta|\ln (T-\lambda)|}\left(\frac{\eta^{2}}{2}-1\right)+o\left(\frac{1}{|\ln (T-\lambda)|}\right) \quad \text { as } \lambda \rightarrow T,
$$

uniformly on compact sets in $\eta$. We next choose $s=s(\lambda)$ as follows:

$$
1=(1-s)|\ln [(T-\lambda)(1-s)]|,
$$

so that

$$
1-s \approx \frac{1}{|\ln (T-\lambda)|} \text { as } \lambda \rightarrow T
$$

Arguing now as in [16, Lemma 6.1] we obtain

$$
u_{\lambda}(\eta, s)=\kappa+\frac{\kappa}{4 \beta} \eta^{2}(1-s)+o(1-s) \text { as } s \rightarrow 1^{-} .
$$


Using (4.4) we have that

$$
\Phi_{\lambda}(\eta, s)=\kappa\left[1+\frac{\beta+1}{4 \beta} \eta^{2}+o(1)\right]^{\gamma}(1-s)^{\gamma} \text { as } s \rightarrow 1^{-} \text {. }
$$

Finally, from (4.5) we get that

$$
[(T-\lambda)(1-s)]^{-\gamma} u(\eta \sqrt{T-\lambda}, \lambda+s(T-\lambda)) \leq \kappa\left[1+\frac{\beta+1}{4 \beta} \eta^{2}+o(1)\right]^{\gamma} \text { as } s \rightarrow 1^{-} \text {, }
$$

and (4.2) follows by setting $T-t=(T-\lambda)(1-s)$.

We next show:

LEMMA 4.2. Under the assumptions of Theorem $A$, for any $R>0$, there exists $C>0$ such that

$$
\left|w_{y}(\eta \sqrt{s}, s)\right| \leq \frac{C}{\sqrt{s}} \quad \text { as } \quad s \rightarrow \infty
$$

uniformly on $|\eta| \leq R$.

Proof. At first we will show that

$$
w(y, s) \geq \kappa-\frac{C}{s},
$$

for $s$ large enough and some positive constant $C$. In view of $(2.10)$ this is equivalent to $v(y, s) \geq-C / s$. From (3.1) and for large enough $s$ we have that

$$
v( \pm 2, s)=\frac{\kappa}{2 \beta s}+o\left(\frac{1}{s}\right)>0, \quad v(0, s)=-\frac{\kappa}{2 \beta s}+o\left(\frac{1}{s}\right)<0 .
$$

We conclude that the (unique) minimum of $v(y, s)$ lies in the interval $(-2,2)$ and (4.9) follows from (3.1).

Once we have obtained (4.9), the proof can be completed in the same way as in [23, Lemma 3.9], that is, by differentiating (2.7) with respect to $y$, multiplying it by sign $\left(w_{y}\right)$, using Kato's inequality and then the variation of constants formula.

We next set

$$
G(y, s)=w^{\beta+1}(y, s)-(\beta+1)
$$

$G(y, s)$ is easily seen to satisfy the equation

$$
G_{s}=G_{y y}-\frac{y}{2} G_{y}+G-L(y, s)
$$

with

$$
L(y, s)= \begin{cases}\beta(\beta+1) w^{\beta-1} w_{y}^{2}, & |y| \leq e^{s / 2} \\ (\beta+1)\left\{\beta w^{\beta-1} w_{y}^{2}-1-w^{\beta} e^{(\gamma-1) s} g(y, s)\right\}, & |y|>e^{s / 2}\end{cases}
$$

In the remainder of this section we will show that

$$
\lim _{s \rightarrow \infty} G(\eta \sqrt{s}, s)=\frac{(\beta+1)^{2}}{4 \beta} \eta^{2},
$$

uniformly for $|\eta| \leq C$. Notice that (4.13) is the same as (4.1) when restated in the original variables. At first we show a preliminary estimate. 
Lemma 4.3. For $s$ large enough there is a positive constant $C$ such that

$$
\|G(\cdot, s)\| \leq \frac{C}{s} .
$$

Proof. For all $y, s$ we know that $w(y, s) \geq B$. If it were true that $w(y, s)<$ $c<+\infty$ then we would have

$$
|G|=\left|w^{\beta+1}-(\beta+1)\right| \leq c|w-\kappa|=c|v|
$$

for some positive $c$, and (4.14) would follow at once from (3.1). But from Lemma 4.1 we have that $w(y, s) \leq c<+\infty$ when $|y| \leq \delta \sqrt{s}$. We may thus write

$$
\|G(\cdot, s)\|^{2}=\int_{|y|<\delta \sqrt{s}}\left|w^{\beta+1}-(\beta+1)\right|^{2} e^{-y^{2} / 4} d y+\int_{|y|>\delta \sqrt{s}}\left|w^{\beta+1}-(\beta+1)\right|^{2} e^{-y^{2} / 4} d y .
$$

By the same reasoning as above we deduce that the first integral is less than or equal to $(C / s)^{2}$, whereas the second one can easily be estimated using the fact that $w(y, s)$ can grow at most quadratically in $y$.

To prove (4.13) we now use the variation of constants formula in (4.11) to obtain

$$
\begin{aligned}
G(y, s)= & E_{1}\left(s, s_{0}\right) \int_{\mathbf{R}} E_{2}\left(y, s ; \lambda, s_{0}\right) G\left(\lambda, s_{0}\right) d \lambda \\
& -\int_{s_{0}}^{s} E_{1}(s, \sigma) \int_{\mathbf{R}} E_{2}(y, s ; \lambda, \sigma) L(\lambda, \sigma) d \lambda d \sigma \\
\equiv & J_{1}-J_{2},
\end{aligned}
$$

where

$$
\begin{gathered}
E_{1}(s, \sigma)=\frac{e^{s-\sigma}}{\sqrt{4 \pi\left[1-e^{-(s-\sigma)}\right]}}, \\
E_{2}(y, s ; \lambda, \sigma)=\exp \left\{-\frac{\left[y e^{-(s-\sigma) / 2}-\lambda\right]^{2}}{4\left[1-e^{-(s-\sigma)}\right]}\right\},
\end{gathered}
$$

and $s, s_{0}$ are related with

$$
s=e^{s-s_{0}} .
$$

We next show:

LEMMA 4.4. There holds

$$
\lim _{s \rightarrow \infty} J_{1}(\eta \sqrt{s}, s)=\frac{(\beta+1)^{2}}{4 \beta} \eta^{2},
$$

uniformly for $|\eta| \leq C$.

Proof. It consists in a slight modification of the proof in [16, Lemma 6.4]. We fix $R>0$ and we split $J_{1}$ into two integrals $J_{1,1}^{R}$ and $J_{1,2}^{R}$ to be performed in the regions $|\lambda| \leq R$ and $|\lambda| \geq R$ respectively. Using (4.14) and standard inequalities one shows that $J_{1,2}^{R}(\eta \sqrt{s}, s)$ tends to 0 as $s, R \rightarrow \infty$ uniformly for $|\eta|<C$. To estimate $J_{1,1}^{R}$ we observe from (3.1) that

$$
G(y, s)=\frac{(\beta+1)^{2}}{4 \beta s}\left(y^{2}-2\right)+g_{R}(y, s),
$$


with $g_{R}(y, s)=o(1 / s)$ as $s \rightarrow \infty$ uniformly for $|y|<R$. Using now (4.19) it can be shown that

$$
J_{1,1}^{R}(\eta \sqrt{s}, s) \rightarrow \frac{(\beta+1)^{2}}{4 \beta} \eta^{2} \text { as } s, R \rightarrow \infty,
$$

uniformly for $|\eta| \leq C$, and (4.18) follows.

To complete the proof of (4.13) we finally have:

LEMMA 4.5. There holds

$$
\lim _{s \rightarrow \infty} J_{2}(\eta \sqrt{s}, s)=0
$$

uniformly for $|\eta| \leq C$.

Proof. Again, minor modifications are required in the proof of [16, Lemma 6.5]. We first break the integral $J_{2}$ into four parts:

$$
\begin{aligned}
J_{2}(y, s)= & \int_{s_{0}}^{s_{0}+A} E_{1} \int_{R<|\lambda|<\delta \sqrt{s_{0}}} E_{2} L d \lambda d \sigma+\int_{s_{0}}^{s_{0}+A} E_{1} \int_{|\lambda| \leq R} E_{2} L d \lambda d \sigma \\
& +\int_{s_{0}+A}^{s} E_{1} \int_{|\lambda| \leq \delta \sqrt{s_{0}}} E_{2} L d \lambda d \sigma+\int_{s_{0}}^{s} E_{1} \int_{|\lambda| \geq \delta \sqrt{s_{0}}} E_{2} L d \lambda d \sigma \\
\equiv & J_{21}+J_{22}+J_{23}+J_{24},
\end{aligned}
$$

where $A$ is such that $s_{0}+A<s-1$. Since $E_{1}, E_{2}$ are known functions (cf. (4.16)) we only need to find estimates for the nonlinear term $L(\lambda, \sigma)$ in the various regions above. Let $C$ denote a positive constant not necessarily the same in each occurrence. (a) For $J_{23}$, recalling (4.12) we have that

$$
L(\lambda, \sigma)=\beta(\beta+1) w^{\beta-1} w_{\lambda}^{2} .
$$

For all $\beta>0$ we have from Lemma 4.2 that $\left|w_{\lambda}(\lambda, \sigma)\right|<C / \sqrt{\sigma}$ for $|\lambda|<\delta \sqrt{s_{0}}$.

- For $\beta>1$, from Lemma 4.1 we have that $|w(\lambda, \sigma)|<C$.

- For $0<\beta \leq 1$, since $w \geq B>0$ we get that $w^{\beta-1} \leq B^{\beta-1}$. Thus, is all cases $|L(\lambda, \sigma)| \leq C / \sigma \leq C / s_{0}$, and one can show that

$$
\left|J_{23}(\eta \sqrt{s}, s)\right| \leq C e^{-A} \text { for }|\eta|<C \text {. }
$$

(b) For $J_{21}$, as in (a) we have that $|L(\lambda, \sigma)| \leq C / s_{0}$. Moreover $\left|y e^{-(s-\sigma) / 2}\right|<C e^{A / 2}$ (for $|\eta|<C$ ), $\left|E_{1}\right| \leq C s_{0}$, and $\left|E_{2}\right| \leq \exp \left\{C e^{A}\right\} e^{-\lambda^{2} / 8}$. It then follows that

$$
\left|J_{21}(\eta \sqrt{s}, s)\right| \leq C A \exp \left\{C e^{A}\right\} e^{-R^{2} / 8} R^{-1} \text { for }|\eta|<C .
$$

(c) For $J_{22}$, we can use Proposition 3.1 to get that $\left|w_{\lambda}\right|<C / \sigma$ (for $|\lambda|<C$ ) and therefore $|L(\lambda, \sigma)|<C / \sigma^{2}$. It then follows that

$$
\left|J_{22}(\eta \sqrt{s}, s)\right| \leq \frac{C}{s_{0}} \text { for }|\eta|<C,
$$

for some $C=C(R, A, \delta)>0$.

(d) Finally, for $J_{24}$ we note that from (4.12) it follows that for $|\lambda|>e^{\sigma / 2},|L(\lambda, \sigma)|<$ $C<+\infty$, whereas for $|\lambda| \leq e^{\sigma / 2}$ we have (see Section 2 ): 
- For $\beta>1,\left|w_{\lambda}\right|$ is bounded and $|w| \leq C(|\lambda|+1)$; therefore $|L(\lambda, \sigma)| \leq$ $C(|\lambda|+1)^{\beta-1}$.

- For $\beta=1$, we claim that

$$
|L(\lambda, \sigma)| \leq C \sigma .
$$

(Let us accept this at the moment and continue.)

- For $0<\beta<1, w^{\beta-1} w_{\lambda}^{2}$ is bounded; therefore $|L(\lambda, \sigma)|<C<+\infty$. In all cases, by arguments similar to those in [16] it can be shown that

$$
\left|J_{24}(\eta \sqrt{s}, s)\right| \rightarrow 0 \text { as } s_{0} \rightarrow \infty, \text { for }|\eta|<C .
$$

In view of (4.21)-(4.24) and by taking $s_{0} \rightarrow \infty, R \rightarrow \infty$, and $A \rightarrow \infty$ in this order, (4.20) follows.

We still have to substantiate our claim (cl). In view of (4.12) this is equivalent to

$$
\left|w_{y}\right| \leq C \sqrt{s} .
$$

Let $t_{*}$ be close enough to $T$ and $u_{*}=\min _{|x| \leq 1} u\left(x, t_{*}\right)$. Set

$$
J(x, t)=\frac{1}{2} u_{x}^{2}-\ln \frac{u}{u_{*}} \quad \text { in } \quad Q_{t_{*}} \equiv(-1,1) \times\left(0, t_{*}\right) .
$$

By a standard argument (quite similar for instance to that in [12, Lemma 3.4]) we get that

$$
\left|u_{x}\right| \leq \sqrt{2 \ln \frac{u}{u_{*}}} \leq \sqrt{2 \ln \frac{1}{u_{*}}} \quad \text { in } Q_{t_{*}} .
$$

Returning now to similarity variables and taking into account (2.9) the result follows.

5. The Quenching Profiles. In this section we will give the proof of Theorem A. The main ingredients in the proof will be a technical Lemma (Lemma 5.1 below) as well as the scaling properties of equation (1.1). We refer to [18] for a more detailed discussion of the ideas involved in the proof. We begin by introducing some notation.

For a given $R>0$, let

$$
\begin{aligned}
& g(s)=(\kappa-\epsilon)(1-s)^{\gamma}, \\
& h(y)=\left\{\begin{array}{c}
(\kappa+\mu \epsilon) \text { if } y \in[-R / 2, R / 2], \\
(\kappa-\epsilon) \text { if } y \in[-R,-R / 2) \cup(R / 2, R] ;
\end{array}\right.
\end{aligned}
$$

where $\mu$ and $\epsilon$ are two positive constants to be specified later. By slightly adapting the arguments in [17, Proposition 3.1] we get

Lemma 5.1. Suppose $z(y, s)$ satisfies equation $(1.1)$ in $Q_{R} \equiv(-R, R) \times(0,1)$ with $z(y, s) \geq g(s)$ in $Q_{R}$ and $z(y, 0) \geq h(y)$ in $[-R, R]$. Then there exists a $\mu>0$ such that, for any $\epsilon>0$ small enough, $z(y, s)$ quenches at most at $y= \pm R$ at time 1. More precisely, there exists a function $F(y)=F(y, R, \mu, \epsilon)$, which is bounded away from zero on compact subsets of $(-R, R)$, and there exists $\lim _{s \uparrow 1} z(y, s)=z(y, 1)$ for $y \in(-R, R)$, and $z(y, s) \leq F(y)$ in $(-R, R) \times(0,1]$.

We are now ready to give the proof of Theorem A.

Proof of Theorem A. Assuming for simplicity that $x_{0}=0$ we will show 


$$
u(x, T)=\left[\frac{(\beta+1)^{2}}{8 \beta}\right]^{\gamma}\left(\frac{|x|^{2}}{|\ln | x||}\right)^{\gamma}(1+o(1))
$$

as $|x| \rightarrow 0$.

Consider the family of functions

$$
\phi_{(t)}(y, s)=(T-t)^{-\gamma} u(\lambda(t)+y \sqrt{T-t}, t+s(T-t)),
$$

where $0<t<T, 0<s<1, \lambda(t)=\eta \sqrt{(T-t)|\ln (T-t)|}$, with $\eta \neq 0$, and

$$
|y \sqrt{T-t}| \leq|\lambda(t)| / 2 \text {. }
$$

Notice that $\phi_{(t)}$ satisfies

$$
\phi_{(t) s}=\phi_{(t) y y}-\phi_{(t)}^{-\beta}
$$

for any fixed $t \in(0, T)$. By (4.9), we have

$$
\phi_{(t)}(y, s) \geq\left[\kappa-\frac{C}{|\ln (T-t)|(1-s)}\right](1-s)^{\gamma} \geq\left[\kappa-\frac{C}{|\ln (T-t)|}\right](1-s)^{\gamma} .
$$

Moreover, it follows from Proposition 4.1 that

$$
\phi_{(t)}(y, 0)=\kappa\left\{1+\left(\frac{\beta+1}{4 \beta}\right)\left[\eta+\frac{y}{\sqrt{|\ln (T-t)|}}\right]^{2}\right\}^{\gamma}+o(T-t)
$$

as $t \uparrow T$. We now claim that for any positive integer $n$ there exist positive constants $c_{n}$ and $C_{n}$ (depending only on $n$ ) such that

$$
0<c_{n} \leq \phi_{(t)}(y, s) \leq C_{n}<+\infty
$$

in $[-n / 2, n / 2] \times[0,1]$, uniformly as $t \uparrow T$. The lower bound in (5.5) follows from (5.3), (5.4), and Lemma 5.1. To see the upper bound we observe that because of assumption $(\mathrm{H}), u_{t}(x, t)$ is nonpositive for all $t \in(0, T)$. Consequently $u(x, t)$ (as well as $\phi_{(t)}(y, s)$, for $t$ fixed) is decreasing with time; therefore

$$
\phi_{(t)}(y, s) \leq \phi_{(t)}(y, 0),
$$

and $\phi_{(t)}(y, 0)$ is bounded above by $(5.4)$.

By a compactness argument and then a diagonal process we see that there is a function $\tilde{\phi}(y, s)$ such that

$$
\phi_{(t)}(y, s) \rightarrow \tilde{\phi}(y, s) \quad \text { as } \quad t \uparrow T
$$

uniformly on compact subsets of $\mathbf{R} \times[0,1]$. Moreover, $\tilde{\phi}$ satisfies

$$
\tilde{\phi}_{s}=\tilde{\phi}_{y y}-\tilde{\phi}^{-\beta} \quad \text { in } \quad \mathbf{R} \times(0,1),
$$

and

$$
\tilde{\phi}(y, 0)=\kappa\left[1+\left(\frac{\beta+1}{4 \beta}\right) \eta^{2}\right]^{\gamma}, \quad y \in \mathbf{R} .
$$

It follows that

$$
\tilde{\phi}(y, s)=\kappa\left[(1-s)+\left(\frac{\beta+1}{4 \beta}\right) \eta^{2}\right]^{\gamma}
$$


Now, set

$$
x=\eta \sqrt{(T-t)|\ln (T-t)|} .
$$

Then we have

$$
|\ln | x|| \approx \frac{1}{2}|\ln (T-t)| \quad \text { as } \quad t \uparrow T
$$

and

$$
x \approx \eta \sqrt{(T-t)} \sqrt{2|\ln | x||} \text { as } t \uparrow T .
$$

Therefore,

$$
T-t \approx \frac{|x|^{2}}{2 \eta^{2}|\ln | x||} \quad \text { as } \quad t \uparrow T .
$$

From (5.6) and (5.9) it follows that

$$
\phi_{(t)}(0,1)=\kappa\left[\left(\frac{\beta+1}{4 \beta}\right) \eta^{2}\right]^{\gamma}+o(1) \quad \text { as } \quad t \uparrow T .
$$

On the other hand, by (5.2),

$$
\phi_{(t)}(0,1)=(T-t)^{-\gamma} u(\eta \sqrt{(T-t)|\ln (T-t)|}, T),
$$

and (5.1) follows from (5.10) and (5.11).

\section{REFERENCES}

[1] S. Angenent, The zero set of a solution of a parabolic equation, J. Reine Angew. Math. 390, 79-96 (1988)

[2] J. Carr, Applications of Centre Manifold Theory, Springer-Verlag, New York, 1981

[3] K. Deng and H. A. Levine, On the blowup of $u_{t}$ at quenching, Proc. Amer. Math. Soc. 106, 1049-1056 (1989)

[4] M. Fila and J. Hulshof, A note on the quenching rate, Proc. Amer. Math. Soc. 112, 473-477 (1991)

[5] M. Fila, J. Hulshof, and P. Quittner, The quenching problem on $N$-dimensional ball, Proceedings of the International Conference on Nonlinear Diffusion Equations and Their Equilibrium States (edited by N. G. Lloyd, J. Serrin, W.-M. Ni, and L. A. Peletier), Gregynog, Wales, August 1989

[6] M. Fila and B. Kawohl, Asymptotic analysis of quenching problems, Rocky Mt. J. Math. 22, 563-577 (1992)

[7] S. Filippas and R. V. Kohn, Refined asymptotics for the blow up of $u_{t}-\Delta u=u^{p}$, Comm. Pure Appl. Math. 45, 821-869 (1992)

[8] A. Friedman and B. McLeod, Blow-up of positive solutions of semilinear heat equations, Indiana Univ. Math. J. 34, 425-447 (1985)

[9] Y. Giga and R. V. Kohn, Asymptoticlly self-similar blow-up of semilinear heat equations, Comm. Pure Appl. Math. 38, 297-319 (1985)

[10] Y. Giga and R. V. Kohn, Characterizing blow-up using similarity variables, Indiana Univ. Math. J. 36, 1-40 (1987)

[11] Y. Giga and R. V. Kohn, Nondegeneracy of blow-up for semilinear heat equation, Comm. Pure Appl. Math. 42, 845-884 (1989)

[12] J.-S. Guo, On the quenching behavior of the solution of a semilinear parabolic equation, J. Math. Anal. Appl. 151, 58-79 (1990)

[13] J.-S. Guo, On the semilinear elliptic equation $\Delta w-\frac{1}{2} y \cdot \nabla w+\lambda w-w^{-\beta}=0$ in $\mathbf{R}^{n}$, Chinese J. Math. 19, 355-377 (1991)

[14] J.-S. Guo, On the quenching rate estimate, Quart. Appl. Math. 49, 747-752 (1991)

[15] J.-S. Guo, The critical length for a quenching problem, Nonlinear Analysis 18, 507-516 (1992)

[16] M. A. Herrero and J. J. L. Velazquez, Blow-up behavior of one-dimensional semilinear parabolic equations, Annales Inst. Henri Poincaré, Sec. C: Analyse Nonlineaire (to appear)

[17] M. A. Herrero and J. J. L. Velazquez, Flat blow-up in one-dimensional semilinear heat equations, Differential Integral Equations 5, 973-997 (1992) 
[18] M. A. Herrero and J. J. L. Velazquez, Blow-up profiles in one-dimensional semilinear parabolic problems, Comm. Partial Differential Equations 17, 205-219 (1992)

[19] M. A. Herrero and J. J. L. Velazquez, Generic behavior of one-dimensional blowup patterns, Ann. Scuola Normale Sup. Pisa (to appear)

[20] H. A. Levine, Quenching, nonquenching, and beyond quenching for solutions of some parabolic equations, Annali di Mat. Pura et Applicata 155, 243-260 (1990)

[21] H. A. Levine, Advances in Quenching, Proceedings of the International Conference on Nonlinear Diffusion Equations and Their Equilibrium States (edited by N. G. Lloyd, J. Serrin, W.-M. Ni, and L. A. Peletier), Gregynog, Wales, August 1989

[22] H. A. Levine, The role of critical exponents in blowup theorems, SIAM Review 32, 262-288 (1990)

[23] J. J. L. Velazquez, Local behavior near blow-up points for semilinear parabolic equations, J. Differential Equations (to appear) 